\title{
Performance Improvement for Hybrid L-band Remote Erbium Doped Fiber Amplifier/Raman using Phase Modulator
}

\author{
Nelidya Md. Yusoff' ${ }^{1}$, A. H. Sulaiman², Sumiaty Ambran ${ }^{3}$, Azura Hamzah $^{4}$, M. A. Mahdi ${ }^{5}$ \\ ${ }^{1}$ Razak School of Engineering \& Advantaged Technology, Universiti Teknologi Malaysia Kuala Lumpur, \\ Jalan Sultan Yahya Petra, 54100 Kuala Lumpur, Malaysia \\ ${ }^{2}$ Institute of Power Engineering, Universiti Tenaga Nasional, Jalan IKRAM-UNITEN, 43000 Kajang, Selangor, Malaysia \\ ${ }^{2,5}$ Wireless and Photonics Networks Research Center, Faculty of Engineering, Universiti Putra Malaysia, \\ 43400 Serdang, Selangor, Malaysia \\ ${ }^{1,3,4}$ Malaysia Jepun International Institute of Technology, Universiti Teknologi Malaysia Kuala Lumpur, \\ Jalan Sultan Yahya Petra, 54100 Kuala Lumpur, Malaysia
}

\begin{tabular}{l}
\hline Article Info \\
\hline Article history: \\
Received Feb 15, 2018 \\
Revised May 2, 2018 \\
Accepted May 9, 2018 \\
\hline
\end{tabular}

Keyword:

Hybrid remote erbium-doped fiber amplifier

L-band

Phase modulation technique SBS suppression

\begin{abstract}
We have demonstrated the performance improvement of L-band hybrid remote Erbium-doped fiber amplifier by introducing a phase modulator to suppress the stimulated Brilloiun scattering (SBS) effect in the transmission. The transmission gain has improved by $12.65 \mathrm{~dB}$ while the noise figure has reduced by $47.1 \mathrm{~dB}$ when $0 \mathrm{dBm}$ signal power is generated at $1590.05 \mathrm{~nm}$ wavelength. Furthermore, the optical signal-to-noise ratio has improved from $7.81 \mathrm{~dB}$ to $29.72 \mathrm{~dB}$ when the signal power is varied from $-30 \mathrm{dBm}$ to $0 \mathrm{dBm}$. By implementing a phase modulator to the input signal somehow able to produce better performance regarding gain, noise figure and optical signalto-noise ratio, especially at the higher signal power as the gain, has been transferred to the Stokes signal and the amplified signal.
\end{abstract}

Copyright $(2018$ Institute of Advanced Engineering and Science. All rights reserved.

\section{Corresponding Author:}

Nelidya Md. Yusoff,

Razak School of Engieering and Advanced Technology,

Universiti Teknologi Malaysia,

Jalan Sultan Yahya Petra, 54200 Kuala Lumpur, Malaysia.

Email: nelidya.kl@utm.my

\section{INTRODUCTION}

Owing to the ever increasing demand and greater bandwidth in telecommunication system leads to the longer distance of transmission distance in optical communication system. However, the effect of attenuation and dispersion in the transmission fiber cannot be eliminated as the signal light propagating through it. This results in some designs on discrete and remote optical amplifier particularly in C-band [1], [2], S-band [3] and L-band [4]-[6] region to overcome this effect. The dispersion effects can be eliminated by introducing dispersion compensating grating, zero dispersion fiber as well as dispersion shifted fiber. These techniques improve the signal performance when it reaches the transmission ends. Hybrid Raman/EDFA is one of the techniques that are used to improve the gain efficiency for the L-band with the integration of Raman amplification in the remote erbium doped fiber amplifier (R-EDFA) [7], [8].

EDFAs have introduced a longer transmission length due to its capabilities of improving the signal gain for even another thousands of kilometers. However, this also will introduce a variety of fibre nonlinearities such as stimulated Brillouin Scattering (SBS) [9], Raman scattering [10] and others. The phase noise will firstly increase drastically and then gradually become flat when the input power is above the SBS threshold. Many methods have been introduced to suppress the SBS such as by using phase modulation [11], fiber Bragg grating [12], using a linearly chirped diode laser [13], and etc. However, phase modulation is among the simplest technique and very effective. 
This paper reported on the use of phase modulator to suppress the SBS effect in the L-band hybrid R-EDFA. A phase modulator with five different tones selection channels has been introduced at the input signal to overcome the SBS effect in the transmission. By using this simple technique, the work have proven that the amplified signal gain has improved significantly as well as the distributed Raman gain and the noise figure.

\section{EXPERIMENTAL SETUP}

Figure 1 shows the experimental setup for remote EDFA using single wavelength dispersion compensating grating (DCG). It consists of Tunable Laser Source (TLS), Raman Pump Unit (RPU), optical spectrum analyzer (OSA) and Optical Power Meter (OPM) as the optical devices involved in this experiment.

While for passive optical components consist of wavelength division multiplexing (WDM), C/L band couplers, L-band isolator, L-band circulators and DCG. An Erbium doped fiber with a numerical aperture of 0.22 , a mode field diameter of $6.2 \mu \mathrm{m}$, EDF with peak absorption coefficient of $39 \mathrm{~dB} / \mathrm{m}$ at $1530 \mathrm{~nm}$ and a cut off wavelength of 923 has been used as the gain medium throughout the experiment. A $41 \mathrm{~km}$ Truewave single mode fiber (SMF) has been used to generate the Raman amplification when the pump light generates from the RPU propagates through it. In this experiment, the pump light is propagated inversely with the signal light generated from the TLS. A $1590.05 \mathrm{~nm}$ signal light will be propagated into the DCG and it will compensate the total dispersion during the light propagation. Futhermore, a phase modulator has been placed just after the TLS to overcome the stimulated Brilloiun scattering effect in the transmission line. The pump light and the signal light will be multiplexed together using $\mathrm{C} / \mathrm{L}$ coupler for double pass amplification when propagating through that EDF. The amplified signal will then be circulated using a circulator into the Raman gain medium before producing the output that is measured using an OSA. The OSA will be used to measure the output performance such as transmission gain, Raman distributed gain, noise figure, optical signal-to-noise ratio and R-EDFA gain.

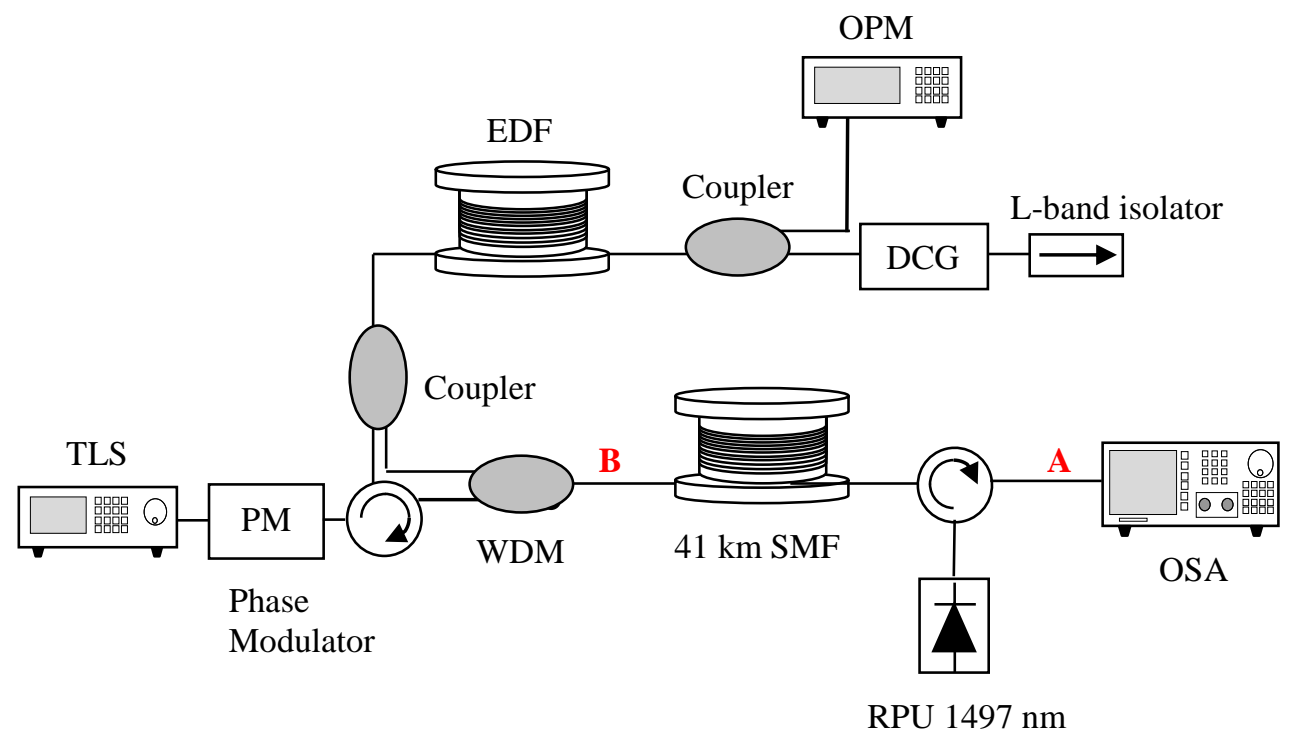

Figure 1. The experimental setup of the remote EDFA using single wavelength dispersion compensating grating.

\section{RESULTS AND DISCUSSIONS}

Figure 2 shows the transmission gain of the amplified signal when the input signal power is varied from $-30 \mathrm{dBm}$ to $0 \mathrm{dBm}$ when the phase modulator is not implemented in the setup. The measurement is taken at Point $\mathrm{A}$ after the signal has gone through the double pass amplification in the EDFA and Raman amplification after propagating through $41 \mathrm{~km}$ SMF. At low input signal which is equal to $-30 \mathrm{dBm}$, the transmission gain obtained is around $41.4 \mathrm{~dB}$ and it keeps reducing as the signal power increases. At the highest signal power, which is around $0 \mathrm{dBm}$, the transmission gain obtained is around $8.5 \mathrm{~dB}$. As can be seen in Figure 2, the gain reduced tremendously as the input signal increases. This is due to the presence of SBS 
effect which is more apparent at high input signal where the gain has been transferred to the Stokes signal and the amplified signal. This finally brings to inaccuracy to the data obtained due to this SBS effects.

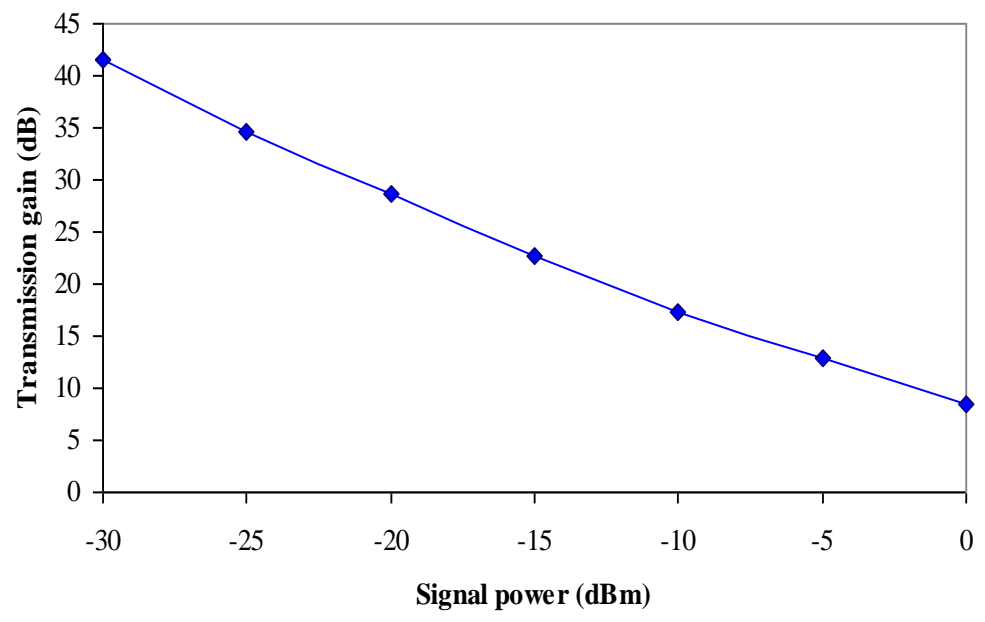

Figure 2. Transmission gain at different signal power with SBS occurrence

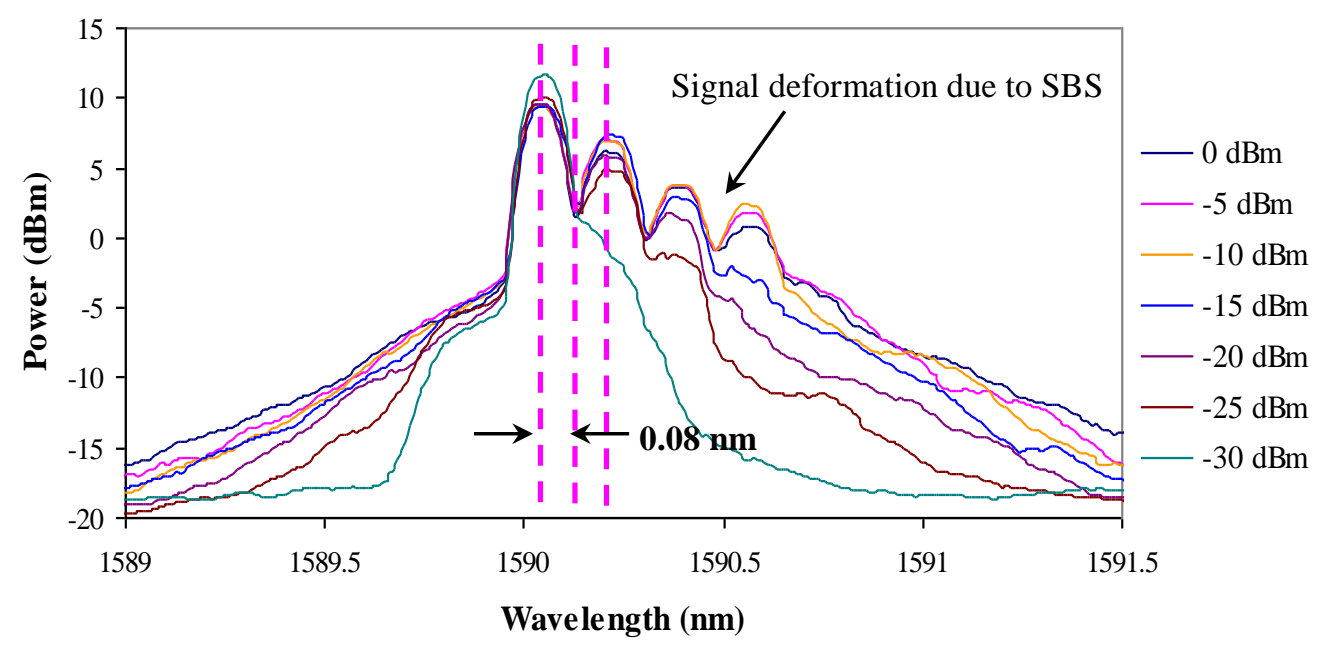

Figure 3. Output spectrum with SBS effect at different signal power

Figure 3 shows the output spectrum that is observed at point A where the SBS effect can be meaningfully observed with $0.1 \mathrm{~nm}$ resolution bandwidth at optical spectrum analyzer. The accumulation of the transmission gain that is contributed by the R-EDFA gain and the Raman amplification contributes to the presence of this SBS effect. The summation of these two components exceeds the threshold for the SBS to take place. The narrow linewidth of the propagating signal also contributed to the occurrence of this SBS. All of these reasons induced the power transferred from the original signal to the first-order Stoke signal which is around $10 \mathrm{GHz}$ or $0.08 \mathrm{~nm}$ spacing. It can be concluded that the SBS effect is more prominent at lower input signal due to the signal amplification.

Figure 4 and Figure 5 show the noise figure (NF) and optical signal-to-noise ratio (OSNR) with the existence of SBS at different signal power. It is clearly can be seen in the graphs have a reverse relationship between each other. The NF starts to increase when the signal power is increased while the OSNR starts to reduce when the signal power is increased. The highest NF of $53.5 \mathrm{~dB}$ is obtained at the highest signal power due to the nonlinearity effect in the fiber. As the NF is increased, this finally brings to the worst OSNR as more noise is added to the amplified signal. When the signal power is equal to OdBm, the OSNR of $6.38 \mathrm{~dB}$ is 
attained. So, in order to abolish the SBS effects in the transmission, phase modulation technique has been introduced to broaden up the linewidth of the input signal by introducing a few frequencies to drive the phase modulator.

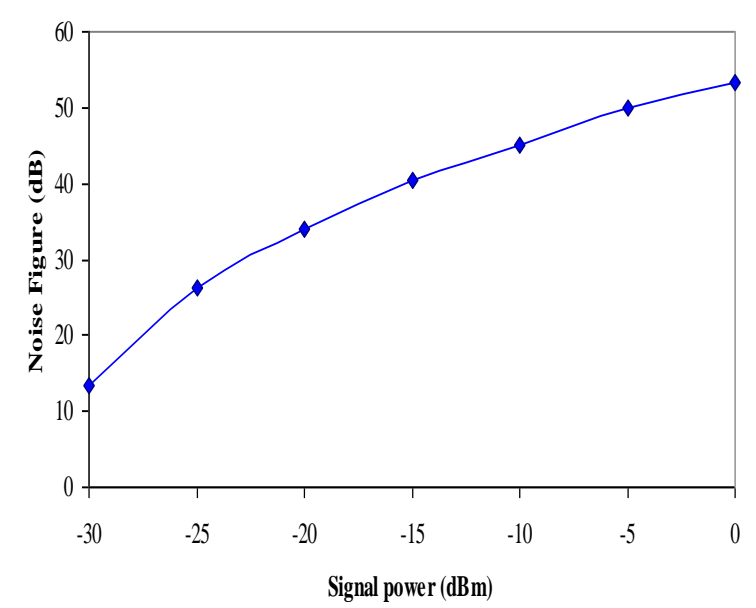

Figure 4. The overall NF when the signal power is varied from $-30 \mathrm{dBm}$ to $0 \mathrm{dBm}$ with SBS occurrence

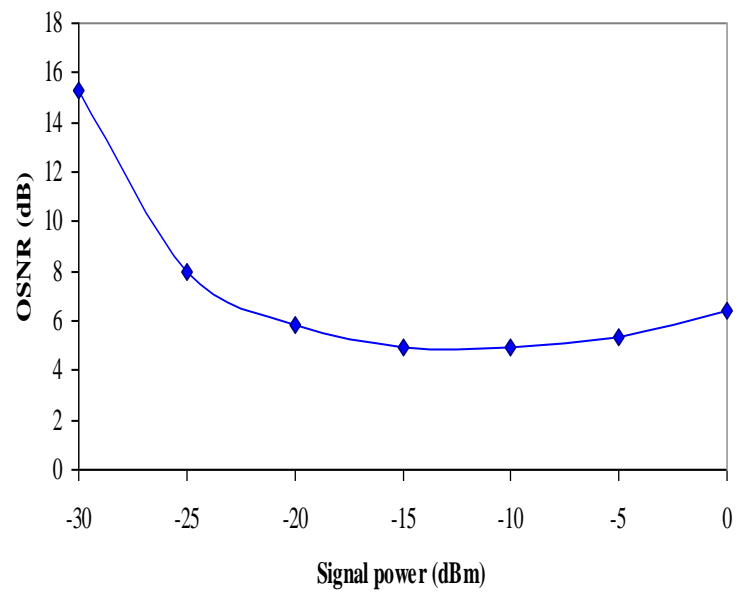

Figure 5. OSNR with the existence of SBS at different signal power

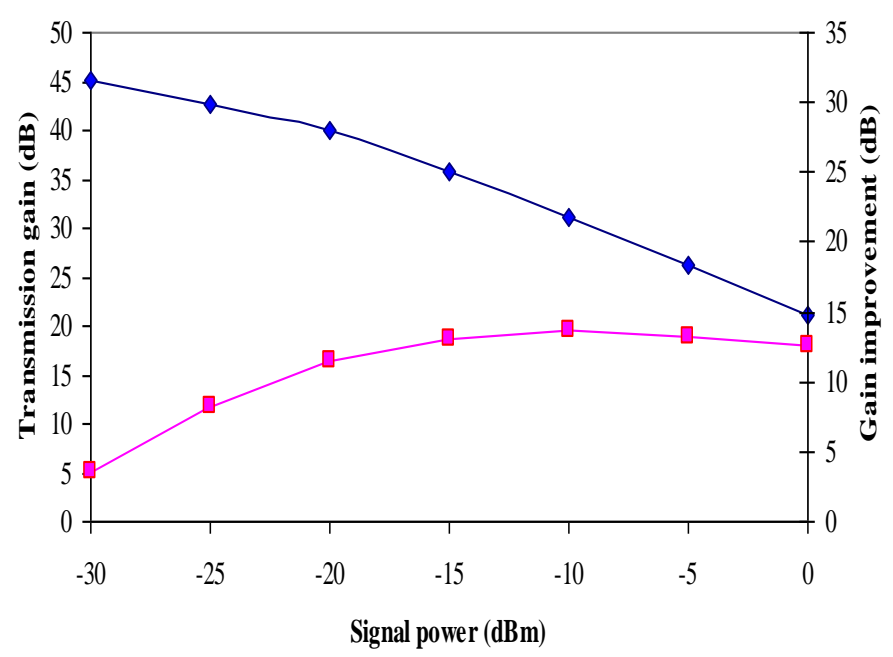

Figure 6. Transmission gain and gain improvement after suppressing the SBS in the transmission line

Referring to Figure 6, it is clear that the gain has improved drastically when a phase modulator is installed in the setup. The transmission gain at $0 \mathrm{dBm}$ signal is around $21.15 \mathrm{~dB}$ and the gain has been improved by $12.65 \mathrm{~dB}$ when the SBS has been suppressed. From the characterization, it is obtained that the SBS threshold power is around $8 \mathrm{dBm}$. When the SBS occurred in the transmission line, a portion of the transmitted light is propagated back in the direction of the transmitter. This result in saturation to optical power at the receiver as well as problems related to back-reflection in the optical signal. So the installation of this phase modulator improved the gain transmission tremendously especially when the input signal is high $(0 \mathrm{dBm})$.

At low input signal which is around $-30 \mathrm{dBm}$, the measured transmission gain is around $45.11 \mathrm{~dB}$ where the gain has been improved by $3.53 \mathrm{~dB}$. Nevertheless, the gain starts to reduce drastically when the signal power goes beyond the input saturation power which is referred to as $-23 \mathrm{dBm}$ due to the saturation effect. 


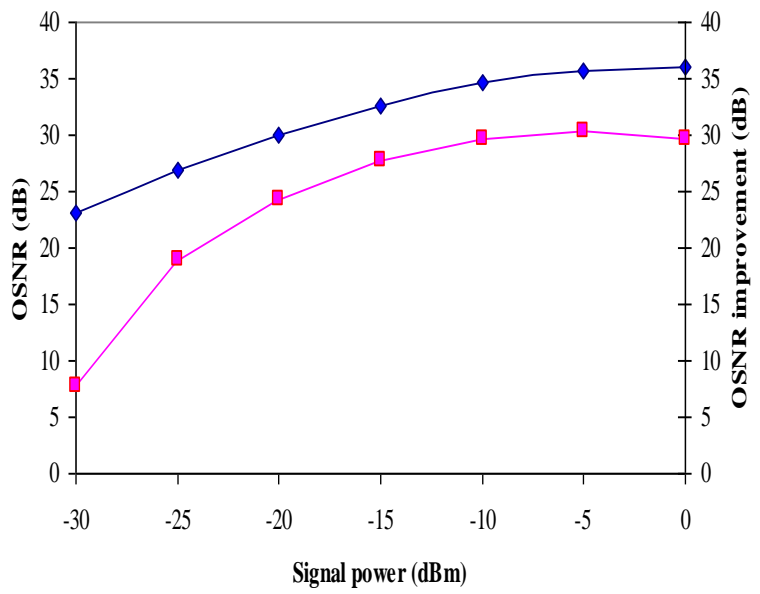

Figure 7. The OSNR with its improvement at different signal power

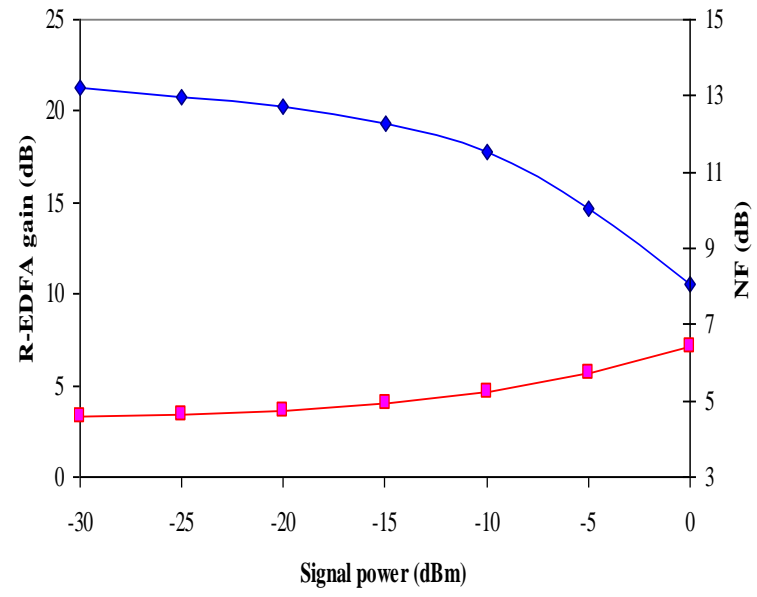

Figure 8 . The R-EDFA and noise figure (NF) in a function of signal power

The OSNR together with its improvement in the function of signal power has been shown in Figure 7. It can be concluded that the OSNR improves enormously when the linewidth of the propagated signal is broadened up using phase modulator in the setup. The OSNR increases progressively from $23.07 \mathrm{~dB}$ to $39.10 \mathrm{~dB}$ when the signal power is varied from $-30 \mathrm{dBm}$ to $0 \mathrm{dBm}$. The OSNR is improved dramatically when PM is applied to the setup where the improvement is from $7.81 \mathrm{~dB}$ to $29.72 \mathrm{~dB}$ when the signal power is varied from $-30 \mathrm{dBm}$ to $0 \mathrm{dBm}$. It can be concluded that the overall performance improves when the propagated signal is free from the SBS effects.

The R-EDFA gain decreased gradually from $21.3 \mathrm{~dB}$ to $18.3 \mathrm{~dB}$ when the input signal is varied from $-30 \mathrm{dBm}$ to $-13 \mathrm{dBm}$ when referring to Figure 8 . This R-EDFA gain is attained at Port $\mathrm{C}$ in the experimental setup. However, when the input signal reaches the saturation point, which is around $-13 \mathrm{dBm}$, the gain starts to reduce extremely to $10.5 \mathrm{dBm}$ at $0 \mathrm{dBm}$ signal. The saturation effect is caused by the higher number of signal photons that contribute to the declining of gain at the higher signal levels. The NF pattern is totally inversed with the gain pattern where the NF keep increasing with the increment of signal power as depicted in Figure 2. When the input signal is at $-30 \mathrm{dBm}$, the NF attained is around $4.6 \mathrm{~dB}$ while when at the highest input signal $(0 \mathrm{dBm})$, the NF obtained is around $6.4 \mathrm{~dB}$. This is due to the supplementary source spontaneous emission that developed together with the propagated signal.

\section{CONCLUSION}

We have demonstrated the performance improvement of L-band hybrid R-EDFA when a phase modulator is introduced to suppress the SBS effect in the transmission. The transmission gain has improved by $12.65 \mathrm{~dB}$ at $0 \mathrm{dBm}$ signal power when the effect of SBS is fully suppressed. At the same time, the noise figure improves tremendously from $53.5 \mathrm{~dB}$ to $6.4 \mathrm{~dB}$ at $0 \mathrm{dBm}$ signal when the linewidth of the amplified signal is broadened up using a phase modulator. Besides having improvement in terms of gain and NF, the OSNR also successfully improved from $7.81 \mathrm{~dB}$ to $29.72 \mathrm{~dB}$ when the signal power is varied from $-30 \mathrm{dBm}$ to $0 \mathrm{dBm}$. By introducing a phase modulator into this L-band hybrid R-EDFA helps to improve the signal power as it reduced the gain competition between the amplified signal and the Stokes signal generated from the SBS effect.

\section{ACKNOWLEDGEMENT}

This research was financially supported by Universiti Teknologi Malaysia under Research University Grant (Vot. No: 15H37) and by Ministry of Higher Education under Fundamental Research Grant Scheme (Vot. No: 4F936).

\section{REFERENCES}

[1] M. Yucel and G. Yenilmez, "The comparison of the gain flattening techniques EDFA configurations in the C/L band," Journal of Optoelectronics and Advanced Materials, vol. 17, pp. 1450-1457, 2015. 
[2] C. Mounia, A. Otman, A. A. Badiaa, C. Fahd, A. A. Vazquez, and E. Y. Mounir, "Gain flatness and noise figure optimization of C-Band EDFA in 16-channels WDM System using FBG and GFF," International Journal of Electrical and Computer Engineering, vol. 7, pp. 289-298, 2017.

[3] K. Kamakshi and V. Rastogi, "Inherently gain flattened S-band erbium doped fiber amplifier based on dual core resonant leaky fiber," Optics Communications, vol. 285, pp. 2821-2825, 2012.

[4] N. M. Yusoff, A. F. Abas, S. Hitam, and M. A. Mahdi, "Bidirectional-pumped L-band erbium-doped fiber amplifier with pump distribution technique," Laser Physics, vol. 22, pp. 1252-1256, 2012.

[5] J. Yang, X. Meng, and C. Liu, "Accurately control and flatten gain spectrum of L-band erbium doped fiber amplifier based on suitable gain-clamping," Optics and Laser Technology, vol. 78, pp. 74-78, 2016.

[6] N. M. Yusoff, A. F. Abas, S. Hitam, and M. A. Mahdi, "Dual-stage L-band erbium-doped fiber amplifier with distributed pumping from single pump laser," Optics Communications, vol. 285, pp. 1383-1386, 2012.

[7] J.-X. Cai, Y. Sun, H. Zhang, H. G. Batshon, M. V. Mazurczyk, O. V. Sinkin, et al., "49.3 Tb/s Transmission Over 9100 km Using C+L EDFA and 54 Tb/s Transmission Over 9150 km Using HybridRaman EDFA," Journal of Lightwave Technology, vol. 33, pp. 2724-2734, 2015.

[8] S. Singh and R. S. Kaler, "Flat-Gain L-Band Raman-EDFA Hybrid Optical Amplifier for Dense Wavelength Division Multiplexed System," IEEE Photonics Technology Letters, vol. 25, pp. 250-252, 2013.

[9] L. Qiliang, Z. Yong, W. Ziyang, Z. Ran, W. Tianshu, and H. Miao, "Theoretical Study on Time Delay of Slow Light by Remodeling SBS Gain and Absorption Spectrum," TELKOMNIKA (Telecommunication, Computing, Electronics and Control) vol. 10, pp. 2442 - 2447, 2012.

[10] W. Dapeng, L. Ning, Y. Aimei, X. Yingxin, and W. Fengyu, "Raman Slow Light in Distributed Raman Fiber Sensors," TELKOMNIKA (Telecommunication, Computing, Electronics and Control) vol. 11, pp. 2442-2447, 2013.

[11] H. S. Pradhan and P. K. Sahu, "Brillouin distributed temperature sensor employing phase modulation and optimization techniques," Optik, vol. 126, pp. 788-794, 2015.

[12] H. Lee and G. P. Agrawal, "Suppression of stimulated Brillouin scattering in optical fibers using fiber Bragg gratings," Optics Express, vol. 11, pp. 3467-3472, 2003.

[13] J. O. White, A. Vasilyev, J. P. Cahill, N. Satyan, O. Okusaga, G. Rakuljic, et al., "Suppression of stimulated Brillouin scattering in optical fibers using a linearly chirped diode laser," Optics Express, vol. 20, pp. 15872-15881, 2012.

\title{
BIOGRAPHIES OF AUTHORS
}

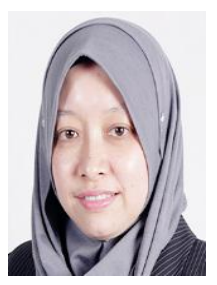

Nelidya Md Yusoff received her Bachelor of Engineering degree majoring in ElectricalTelecommunication from Universiti Teknologi Malaysia in 2002. In 2004, she obtained her MSc in Digital Communication Systems from Loughborough University, United Kingdom. She received her $\mathrm{PhD}$ in Photonics and Fiber Optics System Engineering from Universiti Putra Malaysia in 2013. She is now a senior lecturer at UTM Razak School of Engineering and Advanced Technology. She has served as a reviewer for some reputable journals and until now, she has authored and coauthored over 12 scientific papers in journals and 16 articles in conference proceedings. Her research interest includes discrete and remote Erbium doped fiber amplifier, optical amplifiers, optical sensors and optical communication systems. Currently, she is a member of Institution of Electrical and Electronics Engineers (IEEE) and Optical Society of America as well as the committee for IEEE Photonic Society Malaysia Chapter.

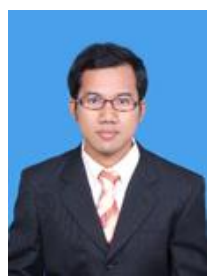

\begin{abstract}
Abdul Hadi Sulaiman received his Bachelor of Science degree majoring Industrial Physics under Department of Physics from Universiti Teknologi Malaysia, Johore in 2007. He obtained his Master of Science (MSc) degree at Universiti Malaya, Malaysia in 2009, under the field of photonics devices for optical communication. In 2015, he completed his Doctor of Philosophy $(\mathrm{PhD})$ degree at Universiti Putra Malaysia, under research area of photonics and fiber optic system engineering. Both research works of his MSc and $\mathrm{PhD}$ were completed at Photonics Research Center and Wireless and Photonics Networks Research Centre, respectively. He also worked as a research assistant from 2012 until 2014 while doing his $\mathrm{PhD}$. After completing his $\mathrm{PhD}$, he served as a senior research officer and postdoctoral fellow at Universiti Teknologi Petronas (2015) and International Islamic University Malaysia (2016), respectively. Currently, he is working at Universiti Tenaga Nasional as a post-doctoral researcher. His research interests include multiwavelength fiber laser, Lyot filter, nonlinear polarization rotation and semiconductor optical amplifier.
\end{abstract}



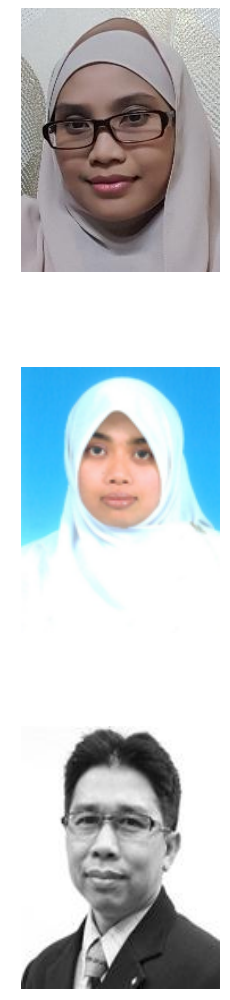

Sumiaty Ambran received her Bachelor's degree in Electronics Telecommunication from the Universiti Teknologi Malaysia, Johor, Malaysia in 2005 and the Master's degree from the Universiti of MARA, Selangor, Malaysia in 2008. She got her Ph.D in Optoelectronics from the Optoelectronics Research Centre (ORC), University of Southampton, United Kingdom in 2013. Currently, she is working with Malaysia-Japan International Institute of Technology (MJIIT), Universiti Teknologi Malaysia. She is actively involved in The Institution of Electrical and Electronics Engineers and a member of the Optical Society of America. Her research interests include planar integrated optical device, optical sensor, optical interconnect, rare-earth-doped optical devices and amplifier application.

Azura Hamzah is a Senior Lecturer at Malaysia-Japan International Institute of Technology (MJIIT), Universiti Teknologi Malaysia. In MJIIT, she is a Full Member for Optical Devices and Systems Ikohza (ODESY) and an Associate Member for Disaster Preparedness and Prevention Centre (DPPC). She received her Bachelor's degree in Communication Engineering from International Islamic University Malaysia, Malaysia in 2005 and the Master's and PhD degree from Universiti Malaya, Malaysia in 2008 and 2012. Her research interests is in optical amplifier and laser, pulse laser, optical sensor, optical interconnect and rare-earth-doped optical devices. Currently, she is a member for The Institution of Electrical and Electronics Engineers and one of the Secretariat for IEEE Photonic Society Malaysia Chapter.

Mohd Adzir Mahdi (M'99-SM'03) received the Bachelor degree with first class honors in Electrical, Electronics and Systems Engineering from the Universiti Kebangsaan Malaysia, Selangor, Malaysia in 1996. Later, he received the Master and Ph.D. degrees with distinctions in Optical Fiber Communications from the Universiti Malaya, Kuala Lumpur, Malaysia in 1999 and 2002, respectively. In January 2003, he joined the Faculty of Engineering, Universiti Putra Malaysia, Selangor, Malaysia where he was an Associate Professor and is now a full Professor. Prior to the current appointment, Adzir was an optical design engineer at IOA Corporation, Sunnyvale, USA and a research officer at Research and Development Division, Telekom Malaysia Berhad. Since 1996, he has been involved in photonics research specializing in optical amplifiers and lasers. He has authored and coauthored over 330 scientific papers in journals and 210 articles in conference proceedings. His research interest includes optical fiber amplifiers and lasers, optical fiber communications, optical sensors and nonlinear optics. Adzir's awards and honors include the IEEE LEOS Graduate-Student Fellowship, the Australia-Malaysia Institute Research Fellowship, the Leading Scientists and Engineers of OIC Member States (COMSTECH), the COMSTECH Young Scientist, the TWAS Young Affiliate Fellow, and the National Academic Award (Young Academician Award). 